\title{
Mean-field and quantum-fluctuation dynamics in the driven dispersive Jaynes-Cummings model
}

\author{
Th. Mavrogordatos ${ }^{1}$, P. Szafulski ${ }^{1}$, E. Ginossar ${ }^{2}$ and M. H. Szymańska ${ }^{1}$ \\ ${ }^{1}$ Department of Physics and Astronomy, University College London, WC1E 6BT, London, UK \\ 2 Advanced Technology Institute and Department of Physics, University of Surrey, GU2 7XH, \\ Guildford, UK
}

\begin{abstract}
In this work we investigate the regime of amplitude bistability in the driven dissipative Jaynes-Cummings (JC) model. We study the semiclassical equation dynamics in contrast to entangled cavity-photon and qubit quantum trajectories, discussing our results in the context of an out-of-equilibrium first order quantum dissipative phase transition for a single JC resonator. Finally, we compare the switching process between metastable states for the two system degrees of freedom by examining a single realization of the random qubit vector in the Bloch sphere next to the intracavity amplitude quasi distributions at given time instants.
\end{abstract}

Keywords: quantum fluctuations, light-matter interaction, first-order quantum phase transition

\section{INTRODUCTION}

The Jaynes-Cummings (JC) model provides a simple basis for describing the interactions between two coupled quantum degrees of freedom: a quantized electromagnetic field and a two-level atom. ${ }^{1}$ In spite of its simplicity, the atom-cavity coupling and the coupling to the driving field induce a strong nonlinearity which is revealed progressively for increasing drive powers. The $\sqrt{n}$ energy dependence is a unique feature of the model and manifests itself both at and off resonance between the two coupled oscillators. ${ }^{2-4}$ A detuning between the qubit transition frequency and the resonator frequency that is much larger than the coupling strength defines the dispersive regime, which is extensively accessed when realizing circuit quantum electrodynamics (circuit QED) experiments with the help of superconducting circuits. ${ }^{5}$ The Hamiltonian describing the interaction of a damped two-level atom (with lowering operator $\sigma_{-}$, raising operator $\sigma_{+}$and inversion operator $\sigma_{z}$ such that $\sigma_{z}=2 \sigma_{+} \sigma_{-}-1$ ) with bare resonant frequency $\omega_{q}$ and a driven damped cavity mode (with photon annihilation and creation operators $a$ and $a^{\dagger}$ respectively) at bare frequency $\omega_{c}$ reads $s^{6,7}$

$$
H_{\mathrm{JC}}=\hbar \omega_{c} a^{\dagger} a+\frac{1}{2} \hbar \omega_{q} \sigma_{z}+i \hbar g\left(a^{\dagger} \sigma_{-}-a \sigma_{+}\right)+i \hbar \varepsilon_{d}\left(a^{\dagger} e^{-i \omega_{d} t}-a e^{i \omega_{d} t}\right),
$$

where $\omega_{d}$ is the driving frequency, $g$ is the atom-cavity coupling strength and $\varepsilon_{d}$ is the driving strength of the external coherent electromagnetic field. The photon loss rate from the cavity is $2 \kappa$ (due to coupling of the system to a zero-temperature reservoir), while the qubit is damped via spontaneous emission at a rate $\gamma$. The dispersive regime is designated by a strong qubit-cavity detuning such that $\delta \equiv\left|\omega_{c}-\omega_{q}\right| \gg g$ and consequently $\lambda \equiv g / \delta \ll 1$. After adding dissipation, the reduced system density matrix obeys the Master Equation $(\mathrm{ME})^{6,7}$

$$
\dot{\rho}=(1 / i \hbar)\left[H_{\mathrm{JC}}, \rho\right]+\kappa\left(2 a \rho a^{\dagger}-a^{\dagger} a \rho-\rho a^{\dagger} a\right)+(\gamma / 2)\left(2 \sigma_{-} \rho \sigma_{+}-\sigma_{+} \sigma_{-} \rho-\rho \sigma_{+} \sigma_{-}\right)
$$

There is a large body of work on the resonant and strong-coupling regime of the driven dissipative JC oscillator, where driving induces a dynamical Rabi splitting due to the photon blockade effect. ${ }^{2}$ Spontaneous dressedstate polarization is related to a second-order quantum phase transition, as it was demonstrated in. ${ }^{8}$ The high excitation strong-dispersive regime features predominantly in the context of quantum nonlinear amplifiers, squeezing associated with the parametric oscillator and the implementation of various non-demolition qubit readout schemes. In our current work we perform a mean-field analysis and compare our results to fluctuationinduced switching between metastable states in the driven-dissipative JC oscillator, which involves two quantum degrees of freedom.

E-mail: t.mavrogordatos@ucl.ac.uk, Telephone: + 44(0)20 76793746

20th Slovak-Czech-Polish Optical Conference on Wave and Quantum Aspects of Contemporary Optics, edited by Jarmila Müllerová, Dagmar Senderáková, Libor Ladányi, L'ubomír Scholtz, Proc. of SPIE Vol. 10142, 101420K · @ 2016 SPIE · CCC code: 0277-786X/16/\$18 · doi: 10.1117/12.2257242

Proc. of SPIE Vol. 10142 101420K-1 


\section{MEAN-FIELD DYNAMICS}

We will now investigate the semiclassical equations of motion in the dispersive regime. Using the ME 2 in a frame rotating with the drive, and factorizing the expectation values of all operator products we arrive at the following set of coupled differential equations:

$$
\begin{gathered}
\frac{d \alpha}{d t}=-\left(\kappa+i \Delta \omega_{c}\right) \alpha-i g m-i \varepsilon_{d}, \\
\frac{d m}{d t}=-\left(\frac{\gamma}{2}+i \Delta \omega_{q}\right) m+i g \alpha \zeta, \\
\frac{d \zeta}{d t}=-\gamma(\zeta+1)+2 i g\left(\alpha^{*} m-m^{*} \alpha\right),
\end{gathered}
$$

where $\alpha=\langle a\rangle, m=\left\langle\sigma_{-}\right\rangle$and $\zeta=\left\langle\sigma_{z}\right\rangle$. In the above semiclassical equations we have defined $\Delta \omega_{c, q}=\omega_{c, q}-\omega_{d}$ are the cavity and qubit detuning parameters from the drive frequency. The mean-field dynamics are dominated by limit cycles and are strongly dependent on the initial conditions. In Fig. 1 we are plotting a trajectory in the phase space for the intracavity field for varying initial conditions of the Bloch vector. As we can observe,
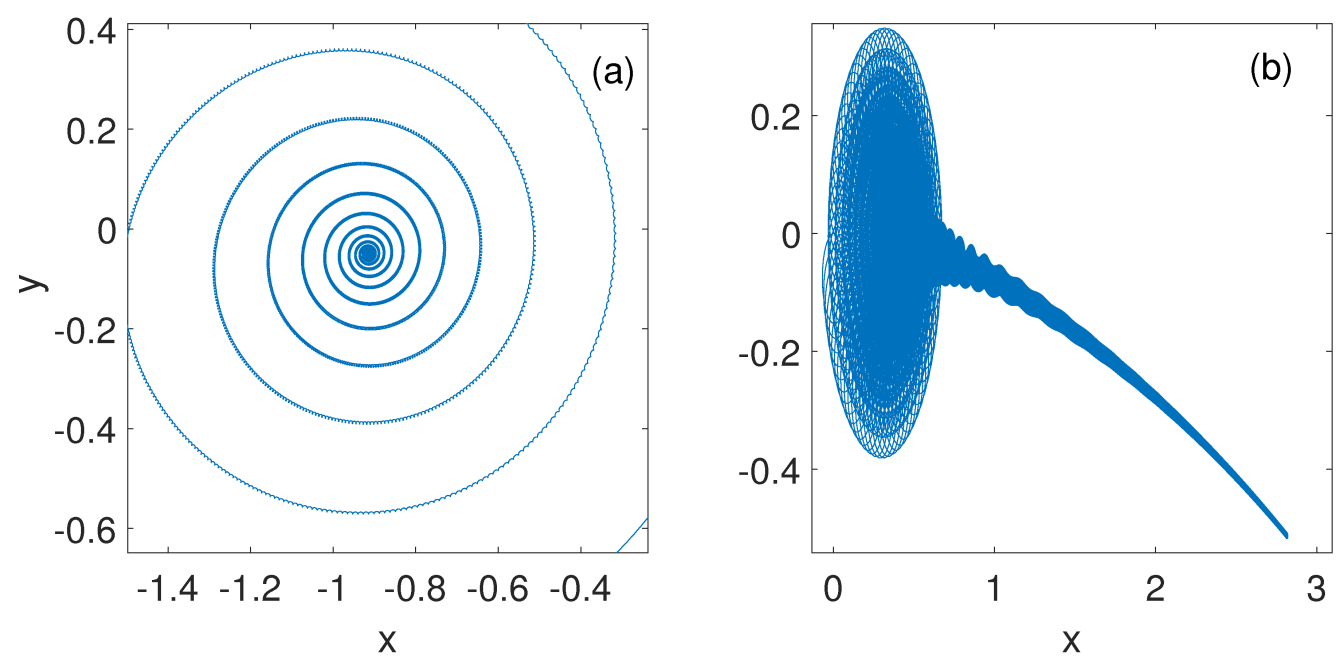

Figure 1. Mean-field equation solutions for different initial conditions. (a) $\alpha(0)=0, m(0)=0$ and $\zeta(0)=-1$, (b) $\alpha(0)=0, m(0)=i / 2$ and $\zeta(0)=0$. Parameters: $\omega_{d} / 2 \pi=10.6100 \mathrm{GHz}, \omega_{c} / 2 \pi=10.5665 \mathrm{GHz}, \omega_{q} / 2 \pi=8.1831 \mathrm{GHz}$, $\gamma /(2 \kappa)=1 / 12, g / \gamma=3347, \varepsilon_{d} / \gamma=100$.

the steady-state mean-field states, one with low mean photon number called 'dim' and one with a higher photon occupation called 'bright', are not globally attracting points but are approached through a limit cycle fashion. In the left panel the initial state is closer to the 'dim' steady state, which is approached in a spiral-like fashion developing progressively a helical pattern. This behaviour is to be compared with the resonant case, where the system stability exhibits a threshold behaviour $\left(2\left|\varepsilon_{d}\right|=g\right) .{ }^{6}$ Above threshold the steady states are foci of limit cycles with frequency $\omega_{\text {cycl }}=\left(4 g\left|\varepsilon_{d}\right| / \kappa\right) \sqrt{1-\left[g /\left(2\left|\varepsilon_{d}\right|\right)\right]^{2}}$. In our case, with an a priori detuning between the drive, cavity and qubit we do not expect a threshold behaviour for weak system excitations, close to the critical point $C_{1}$ from where amplitude bistability emerges. ${ }^{3}$

\section{FORMULATION OF STOCHASTIC SCHRÖDINGER EQUATIONS}

The effect of quantum fluctuation switching can be illustrated by invoking the concept of a Markov piecewise deterministic process (PDP) unravelling the Lindblad ME we have defined in the previous section. The dynamical evolution of the open system is formulated in terms of a stochastic process $\psi(t)$ in the underlying Hilbert space. 
The Liouville ME describing the evolution of the density functional $P[\psi, t]$ (with the formal correspondence $\left.\rho_{\mathrm{s}}\left(x, x^{\prime}, t\right)=\int P[\psi, t] \psi(x) \psi^{*}\left(x^{\prime}\right) d \psi, d \psi^{*}\right) \operatorname{reads}^{9}$

$$
\begin{aligned}
\frac{\partial}{\partial t} P[\psi, t]=i \int & {\left[\frac{\delta}{\delta \psi(x)} G(\psi)(x)-\frac{\delta}{\delta \psi^{*}(x)} G(\psi)^{*}(x)\right] P[\psi, t] d x } \\
& +\int\{W[\psi \mid \bar{\psi}] P[\bar{\psi}, t]-W[\bar{\psi} \mid \psi] P[\psi, t]\} d \bar{\psi} d \bar{\psi}^{*}
\end{aligned}
$$

The first term in the above equation describes the deterministic evolution and the second one reflects the stochastic nature of the process (quantum jumps). The generator of the deterministic evolution time intervals reads

$$
G(\psi)=\tilde{H} \psi+i \kappa\|a \psi\|^{2}+i \frac{\gamma}{2}\left\|\sigma_{-} \psi\right\|^{2}
$$

where

$$
\tilde{H}=H_{\mathrm{JC}}-i \kappa a^{\dagger} a-i \frac{\gamma}{2} \sigma_{+} \sigma_{-}
$$

is a non-Hermitian operator giving rise to non-unitary time evolution as a consequence of the quantum jumps initiated by the Lindblad operators $a$ and $\sigma_{-}$featuring in the Markovian ME. With increasing time during the coherent evolution of the wavefunction, its norm decays and a jump to a new state becomes increasingly likely. Given that a jump occurred at time $t$ and resulted in the wavefunction $\bar{\psi}$, the probability for the next jump to take place somewhere in the interval $(t, t+\tau)$ is $p_{\mathrm{J}}[\bar{\psi}, t]=1-\|\exp (-i \tilde{H} \tau) \bar{\psi}\|^{2}$.

The transition functional (stochastic evolution), corresponding to a jump from the anterior state $\bar{\psi}$ to the final state $\psi$, assumes the form

$$
W[\psi \mid \bar{\psi}]=2 \kappa\|a \bar{\psi}\|^{2} \delta\left(\frac{a \bar{\psi}}{\|a \bar{\psi}\|}-\psi\right)+\gamma\left\|\sigma_{-} \bar{\psi}\right\|^{2} \delta\left(\frac{\sigma_{-} \bar{\psi}}{\left\|\sigma_{-} \bar{\psi}\right\|}-\psi\right) .
$$

Assuming now many infinitesimal jumps, in contrast to discontinuous jumps generating derivatives of all orders, leads to the so-called diffusion approximation which can be described by a Fokker-Planck equation. The process concludes with the numerical solution of a stochastic Scrödinger equation of the following Itô form with a real Wiener increment $d W(t)$

$$
d \psi(t)=D_{1}[\psi(t)] d t+D_{2}[\psi(t)] d W(t)
$$

with the deterministic (drift) term

$$
D_{1}[\psi]=-i H_{\mathrm{JC}}+\kappa\left(\left\langle a+a^{\dagger}\right\rangle_{\psi} a-a^{\dagger} a-\frac{1}{4}\left\langle a+a^{\dagger}\right\rangle_{\psi}^{2}\right) \psi+\frac{\gamma}{2}\left(\left\langle\sigma_{-}+\sigma_{+}\right\rangle_{\psi} \sigma_{-}-\sigma_{+} \sigma_{-}-\frac{1}{4}\left\langle\sigma_{-}+\sigma_{+}\right\rangle_{\psi}^{2}\right) \psi
$$

and the stochastic (diffusion) term

$$
D_{2}[\psi]=\sqrt{2 \kappa}\left(a-\frac{1}{2}\left\langle a+a^{\dagger}\right\rangle_{\psi}\right) \psi+\sqrt{\gamma}\left(\sigma_{-}-\frac{1}{2}\left\langle\sigma_{-}+\sigma_{+}\right\rangle_{\psi}\right) \psi
$$

In the above expressions we have used the notation $\langle C\rangle_{\psi} \equiv\langle\psi|C| \psi\rangle$. Under this formalism we estimate the expectation value $M_{t} \equiv \mathbf{E}(\langle\psi(t)|C| \psi(t)\rangle)=\int\langle\psi|C| \psi\rangle P[\psi, t] d \psi d \psi^{*}$ of a system operator $C$ by the quantity

$$
\hat{M}_{t}=\frac{1}{K} \sum_{k=1}^{K}\left\langle\psi^{k}(t)|C| \psi^{k}(t)\right\rangle,
$$

where $k$ denotes the different realizations. In our work we use the explicit second order weak scheme approximating $\psi\left(t_{n}\right)$ by $\psi_{n}(n=1,2 \ldots T / \Delta t)$ such that $\mathbf{E}\left[\left\|\psi_{n}-\psi\left(t_{n}\right)\right\|\right] \leq \Delta t^{2}$ (weak convergence), where $\Delta t=T / N$ 
is the time step. This method avoids the determination and evaluation of derivatives of various orders of the drift and the diffusion coefficients (unlike the Taylor schemes). According to the scheme proposed by Platen for scalar noise: ${ }^{10}$

$\psi_{n+1}=\psi_{n}+\frac{1}{2}\left(D_{1}\left[\bar{y}_{n}\right]+D_{1}\left[\psi_{n}\right]\right) \Delta t+\frac{1}{4}\left(D_{2}\left[\bar{y}_{n}^{+}\right]+D_{2}\left[\bar{y}_{n}^{-}\right]+2 D_{2}\left[\psi_{n}\right]\right) \Delta W+\frac{1}{4}\left(D_{2}\left[\bar{y}_{n}^{+}\right]-D_{2}\left[\bar{y}_{n}^{-}\right]\right)\left[\Delta W^{2}-\Delta t\right] \Delta t^{-1 / 2}$,

where $\bar{y}_{n}=\psi_{n}+D_{1}\left[\psi_{n}\right] \Delta t+D_{2}\left[\psi_{n}\right] \Delta W$ and $\bar{y}_{n}^{ \pm}=\psi_{n}+D_{1}\left[\psi_{n}\right] \Delta t \pm D_{2}\left[\psi_{n}\right] \sqrt{\Delta t}$. Here, $\Delta W$ is a random variable with a Gaussian distribution of zero mean and variance $\Delta t$. The above formulation allows us to track the time evolution of a pure state and calculate the average value of the system observables using the time varying density matrix $\rho(t)=|\psi(t)\rangle\langle\psi(t)|$ in the steady state.

\section{THE DUFFING OSCILLATOR NONLINEARITY}

We will now visit the Kerr effect, which is the starting point of the nonlinearity exhibited by the driven dispersive JC oscillator. ${ }^{3,11}$ After applying the dispersive unitary transformation for diagonalizing the system Hamiltonian, the operator 1 following normal ordering assumes the form (up to quartic order in the small parameter $\lambda=g / \delta$ )

$$
H_{\mathrm{D}} \simeq \hbar\left(\omega_{c}+\chi^{\prime}\right) a^{\dagger} a+\hbar\left[\omega_{q}+2 \chi\left(a^{\dagger} a+\frac{1}{2}\right)\right] \frac{\sigma_{z}}{2}+\hbar \chi^{\prime}\left(a^{\dagger} a\right)^{2} \sigma_{z},
$$

where $\chi=g^{2}\left(1-\lambda^{2}\right) / \delta$ and $\chi^{\prime}=-g^{4} / \delta^{3}$. The above Duffing oscillator approximation is valid if $4 \lambda^{2} N \ll 1$, where $N$ stands for the total number of the system excitations. The corresponding Wigner distribution function takes the form of the following series expansion, which explicitly demonstrates the build-up of nonlinearity for increasing drive strength:

$$
W\left(\alpha, \alpha^{*}\right)=2 \frac{e^{-2|\alpha|^{2}}}{\pi_{0} F_{2}\left(c, c^{*}, 2\left|\tilde{\varepsilon}_{d}\right|^{2}\right)}\left|1+\frac{z}{D_{1}}+\frac{z^{2}}{2 D_{2}}+\ldots\right|^{2}
$$

where $z=\sqrt{-8 \tilde{\varepsilon}_{d} \alpha^{*}},{ }_{0} F_{2}$ is a generalized hypergeometric function and $D_{n}=c \cdot(c+1) \ldots \cdot(c+n-1)$. In the above expressions $\tilde{\varepsilon}_{d}=\varepsilon_{d} /\left(i \chi^{\prime} \sigma_{z}\right)$ and $c=\left\{\kappa+i\left[\omega_{c}-\omega_{d}-\left(g^{2} / \delta\right) \sigma_{z}+\mathcal{O}\left(g^{4} / \delta^{3}\right)\right]\right\} /\left(i \chi^{\prime} \sigma_{z}\right)$ with a fixed $\sigma_{z}=\left\langle\sigma_{z}\right\rangle=-1$. Based on Eq. 17 we can calculate the (modulus of) the first moment $|\langle a\rangle|$ for varying frequency and drive strength, in a similar fashion to, ${ }^{11}$ which exhibits the characteristic coherent cancellation dip as opposed to the square root of the photon number $\sqrt{\left\langle a^{\dagger} a\right\rangle}$.

In Fig. 2 we are comparing the development of intracavity nonlinearity between the Duffing approximation and the results obtained by solving numerically the quantum ME for increasing driving strength in the region of weak bistability (in the phase space with $\alpha=x+i y$ ). The Wigner functions show a clear departure from the Gaussian form that describes a coherent state. In this regime, the qubit does not participate significantly in the system nonlinearity after having dressed the cavity with the corresponding quartic order terms that feature in Eq. 16. The excitation path evidences the formation of a spiral that we will encounter later on in the regime of the full JC oscillator bistability. The approximation of Eq. 16 breaks down as the qubit becomes more entangled to the cavity and renormalizes the drive in the ME following the dispersive transformation.

\section{CAVITY FIELD NEXT TO THE BLOCH SPHERE}

We will now proceed to the region of the full JC nonlinearity where the Duffing approximation is not applicable and the qubit degrees of freedom cannot be treated effectively as constants of motion. In Fig. 3 we are juxtaposing the $Q$-function for the cavity field in the steady state, $Q(x+i y)=\left\langle x+i y\left|\rho_{\mathrm{c}, \mathrm{ss}}\right| x+i y\right\rangle$ extracted from the ME, to the qubit vector projected onto the equatorial plane of the Bloch sphere for a single quantum trajectory in the steady state. Each point corresponds to a single time instant along the trajectory. As we can observe, both distributions capture the two squeezed semi-coherent metastable states. The scatter we observe in Fig. 3 (b) is due to quantum fluctuations responsible for the switching between the two metastable states and the decay 

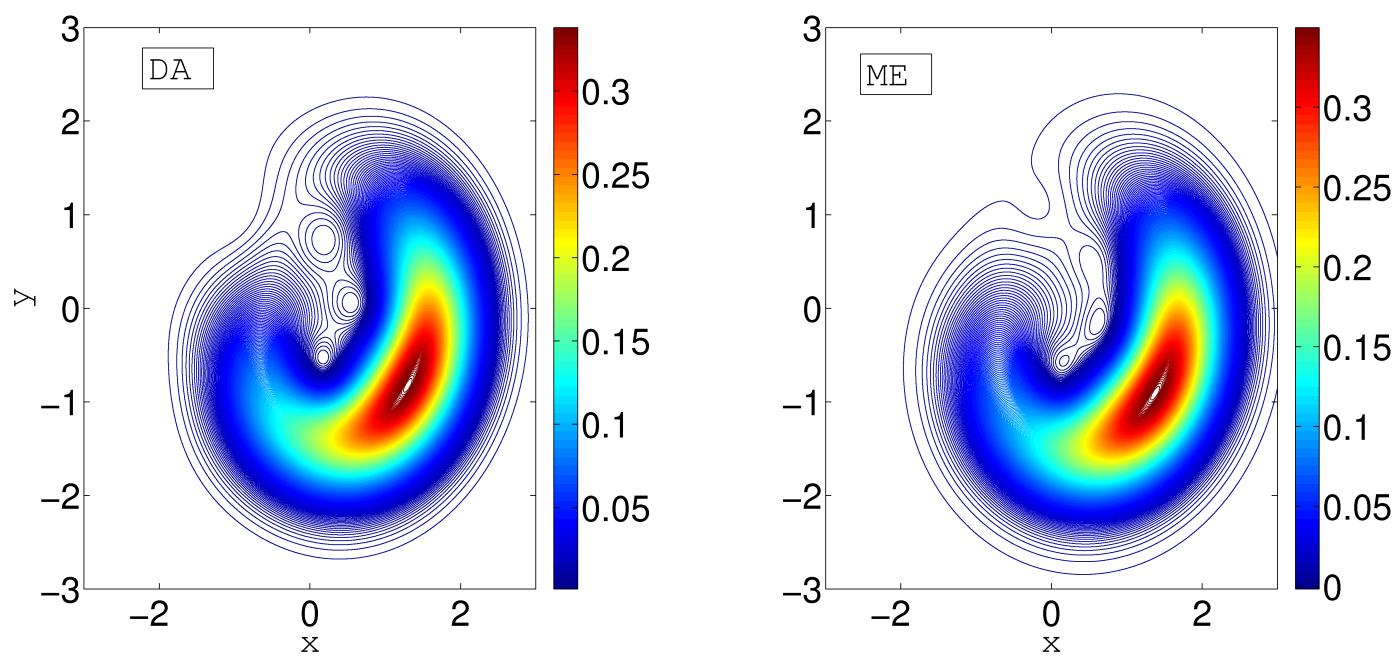

Figure 2. The Kerr nonlinearity. Wigner distributions in the region of $C_{1}$ extracted from the Duffing approximation (DA) and the full JC Master Equation (ME). Parameters: $\omega_{d} / 2 \pi=10.6100 \mathrm{GHz}, \omega_{c} / 2 \pi=10.5665 \mathrm{GHz}, \omega_{q} / 2 \pi=8.1831 \mathrm{GHz}$, $\gamma /(2 \kappa)=1 / 12, g / \gamma=3347$.
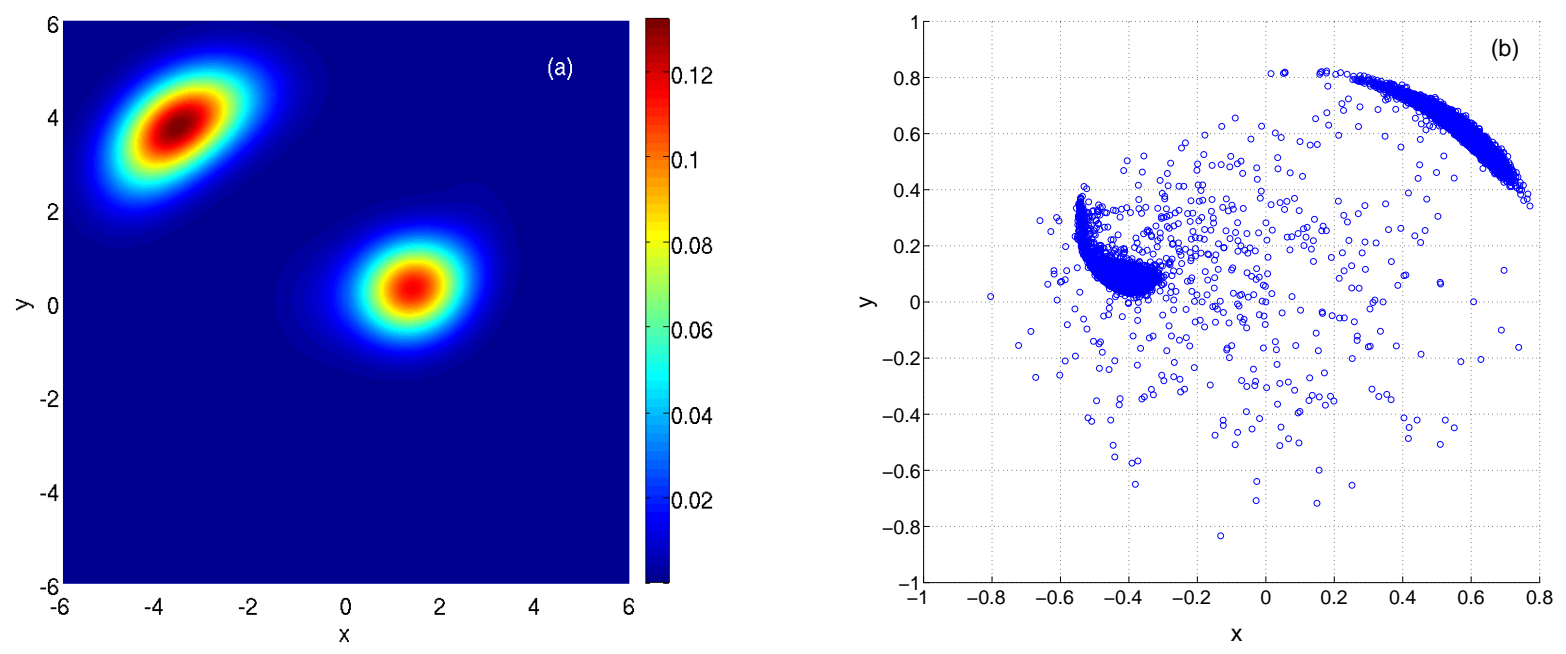

Figure 3. Cavity and qubit distributions. (a) Intracavity joint quasi-probability $Q$-function $Q(x+i y)$ for $\omega_{d} / 2 \pi=10.5720$ $\mathrm{GHz}$ showing two peaks corresponding to two semi-coherent states, indicating the presence of bimodality (b) Equatorial plane projection of the Bloch sphere for a single quantum trajectory. Parameters: $\omega_{c} / 2 \pi=10.5665 \mathrm{GHz}, \omega_{q} / 2 \pi=$ $10.1831 \mathrm{GHz}, \gamma /(2 \kappa)=1 / 12, g / \gamma=600, \varepsilon_{d} /(2 \kappa)=43 / 12$.

of the unstable mean-field state. Fig. 3 indicates an excitation path where the qubit participates now very actively in the switching for the selected driving parameters. Squeezing of the qubit distribution is an evidence of nonlinearity and corresponds to the squeezing along the mean-field quadrature we observe for the intracavity field. The qubit inversion and the intracavity field exhibit simultaneous bistability in the steady state, following 
the mean-field equations

$$
\alpha_{\mathrm{ss}}=-\frac{i \varepsilon_{d}}{\kappa+i \Delta \omega_{c}}\left[1+\frac{2 C\left(1+i \Delta \omega_{c} / \kappa\right)^{-1}\left(1+2 i \Delta \omega_{q} / \gamma\right)^{-1}}{1+\frac{n}{n_{0}\left(1+4 \Delta \omega_{q}^{2} / \gamma^{2}\right)}}\right]^{-1}
$$

and

$$
\zeta_{\mathrm{ss}}=-\left[1+\frac{n}{n_{0}\left(1+4 \Delta \omega_{q}^{2} / \gamma^{2}\right)}\right]^{-1} .
$$

In the above expressions, $n$ is the intracavity photon number, $C=g^{2} /(\kappa \gamma)$ is the co-operativity parameter and $n_{0}=\gamma^{2} /\left(8 g^{2}\right)$ is the saturation photon number, the scale parameter of a saturable absorber at resonance. In this case we can use the expression to which Eq. 18 reduces for $\Delta \omega_{c}=\Delta \omega_{q}=0$, in order to appreciate the onset of bistability (for $C>4)^{12}$

$$
Y=X\left[1+\frac{2 C}{1+X^{2}}\right]
$$

with the scaled variables $X=n_{0}^{-1 / 2}\langle a\rangle$ and $Y=n_{0}^{-1 / 2}\left(\varepsilon_{d} / \kappa\right)$. The photon number $n_{0} X^{2}$ in the semiclassical states tends to infinity for $n_{0} \rightarrow \infty$ (good-cavity limit) and to zero for $n_{0} \rightarrow 0$ (bad-cavity limit). In the strongly dispersive regime, under the hierarchy of scales $\gamma \ll 2 \kappa \ll g^{2} / \delta \ll \delta \ll \omega_{c}$, a similar rôle is played by the parameter $n_{d}=4 \lambda^{2}$ : as $\lambda \rightarrow 0$ semiclassical bistability involves very large photon numbers, while for $\Delta \omega_{c}=0$ the photon number is set by the ratio $\left|\varepsilon_{d} / \kappa\right|^{2}{ }^{3}$

The smallness of the parameter $n_{d}$ allows the expansion of the square root in the dispersive transformation to yield Eq. 16 in the region of relatively weak nonlinearity. The corresponding limit is one of a weak coupling in the sense that the appearance of nonlinearity requires a large photon number as $g \rightarrow 0 .^{2}$
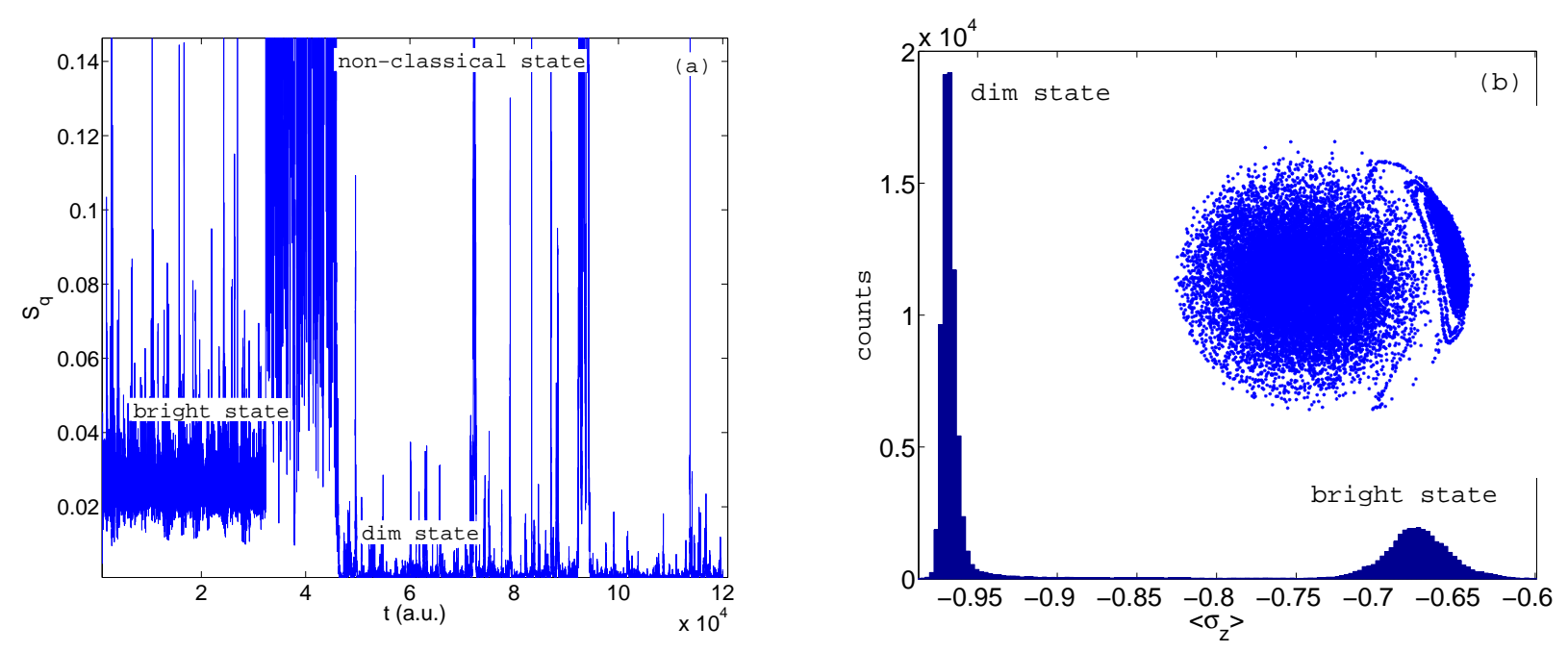

Figure 4. Single quantum trajectory: dynamics and statistics. (a) Entanglement entropy for $\omega_{d} / 2 \pi=10.6005 \mathrm{GHz}$ calculated for the reduced qubit matrix via the relation $S_{q}=-\operatorname{tr}\left(\rho_{\mathrm{ss}, \mathrm{q}} \ln \rho_{\mathrm{ss}, \mathrm{q}}\right)$. (b) Histogram for the average qubit inversion $\left\langle\sigma_{z}\right\rangle$. The inset shows the equatorial plane projection in the Bloch sphere with the bright state distribution on the right being approached via a limit cycle. Parameters: $\omega_{c} / 2 \pi=10.5665 \mathrm{GHz}, \omega_{q} / 2 \pi=8.1831 \mathrm{GHz}, \gamma /(2 \kappa)=1 / 12$, $g / \gamma=3347, \varepsilon_{d} /(2 \kappa)=100 / 12$.

As we can deduce from Fig. 4 the entanglement of the two degrees of freedom is different in the two metastable states and increases with increasing system excitation $N=a^{\dagger} a+\sigma_{+} \sigma_{-}$. It is also interesting to note that the 
von Neumann entropy is significantly larger during the occupation period of the non-mean-field transient state. The inset of Fig. 4 depicts the equatorial projection of the Bloch sphere with the bright state distribution being clearly separated. Fig. 5 details the switching process through a change in the mixture of coherent states that
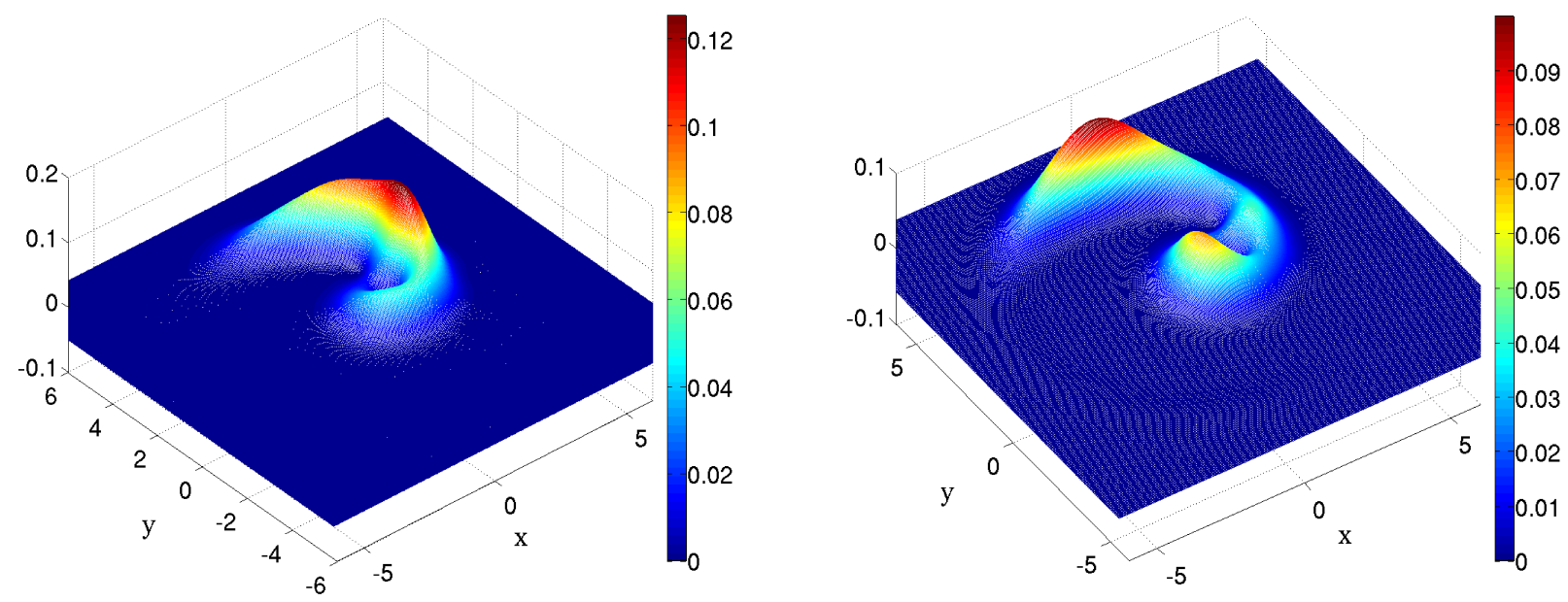

Figure 5. Decay of the unstable state during a switch to the bright state. Joint quasi-probability $Q$-function $Q(x+i y)$ for $\omega_{d} / 2 \pi=10.6005 \mathrm{GHz}$ in a single quantum trajectory leading to an 'up' switch for two given time instants. The process shows the decay of the unstable semiclassical state due to quantum fluctuations. The distribution on the left corresponds to an earlier time. Parameters: $\omega_{c} / 2 \pi=10.5665 \mathrm{GHz}, \omega_{q} / 2 \pi=8.1831 \mathrm{GHz}, \gamma /(2 \kappa)=1 / 12, g / \gamma=3347$, $\varepsilon_{d} /(2 \kappa)=100 / 12$.

comprise the intracavity field distribution. The unstable mean-field state, which does not appear in the averaged Q-function distributions in the steady state, decays and gives its place to a switch to the bright metastable state. Switching events take place along an excitation spiral, owning to the detuning between the qubit and the resonant cavity mode.

\section{CONCLUSIONS}

In this paper we have examined the effect of quantum fluctuations when studying the switching between metastable states in the strongly driven dispersive Jaynes-Cummings model. This is an example of a quantum dissipative oscillator where coupling to the environment plays a major rôle in the formation of macroscopic states. We have used both the exact numerical solution of the Markovian Master Equation and single quantum trajectories to which the former is unravelled. We have presented distributions in the Bloch sphere in equal footing to quasiprobability functions for the intracavity amplitude and showed two metastable mean-field states between which quantum fluctuation switching takes place. As the two quantum degrees of freedom are coupled, the (bipartite) entanglement entropy varies significantly during the switching process.

\section{ACKNOWLEDGMENTS}

Th. M. wishes to thank H. J. Carmichael for helpful discussions and P. Brookes for his contribution in the numerical simulations of SSEs. Th. M. and M. H. S. acknowledge support from the Engineering and Physical Sciences Research Council (EPSRC) under grants EP/I028900/2 and EP/K003623/2.

\section{REFERENCES}

[1] E. T. Cummings and F. W. Jaynes, "Comparison of Quantum and Semiclassical Radiation Theories with Application to the Beam Maser", Proc. IEEE 51, pp.89-109, 1963. 
[2] H. J. Carmichael, "Breakdown of Photon Blockade: A Dissipative Quantum Phase Transition in Zero Dimensions", Phys. Rev. X 5, pp.031028-(1-16), 2015.

[3] L. S. Bishop, E. Ginossar and S. M. Girvin, "Response of the Strongly Driven Jaynes-Cummings Oscillator", Phys. Rev. Lett. 105, pp. 100505(1-4), 2010.

[4] J. M. Fink, M. Göpl, M. Baur, R. Bianchetti, P. J. Leek, A. Blais and A. Wallraff, "Climbing the JaynesCummings Ladder and Observing its Nonlinearity in a Cavity QED System", Nature (London), 454, pp. 315-318, 2008.

[5] M. Boissonneault, J. M. Gambetta and A. Blais, "Dispersive regime of circuit QED: Photon-dependent qubit dephasing and relaxation rates", Phys. Rev. A 79, pp.013819(1-17), 2009.

[6] H. J. Carmichael, Statistical Methods in Quantum Optics 2, Non-Classical Fields, Springer, Berlin, 2008.

[7] D. F. Walls and G. J. Milburn, Quantum Optics, Springer (2nd Edition), Berlin, 2010.

[8] P. Alsing and H. J. Carmichael, "Spontaneous dressed state polarization of a coupled atom and cavity mode", Quantum Opt. 13, pp.13-32, 1991.

[9] H. P. Breuer and F. Petruccione, The Theory of Open Quantum Systems, Oxford University Press, Oxford, 2002.

[10] P. E. Kloeden and E. Platen, Numerical Solution of Stochastic Differential Equations, Springer, Berlin, 1992.

[11] P. D. Drummond and D. F. Walls, "Quantum theory of optical bistability. I: Nonlinear polarisability model", J. Phys. A: Math. Gen. 13, pp.725-741, 1980.

[12] C. M. Savage and H. J. Carmichael, "Single Atom Optical Bistability", IEEE Journal of Quantum Electronics 24, pp.1495-1498, 1988. 